\title{
A Study on Attrition among New Entrants in Software Testing Professionals
}

\author{
M. Kannan \\ Research Scholar, Department of Computer \\ Science and Applications \\ SCSVMV University, Enathur, \\ Kanchipuram-631561
}

\author{
K. Vivekanandan \\ Professor, Department of Computer Science and \\ Engineering \\ Pondicherry Engineering College, \\ Puducherry - 605014
}

\begin{abstract}
The Purpose of the Study was to investigate the turnover intentions among new entrants in software testing professionals who are working in software industry which is located in Chennai. The Samples unit $[\mathrm{N}=135$, Male $=67$, Female $=68]$ was drawn from Software Industry. The Study investigated the Organizational Satisfaction, Job Satisfaction, Interpersonal Relationship with Supervisor's and Life Satisfaction has significant impact on Turnover Intentions among new entrants. The results were interpreted using SPSS 17.0 and the findings given for the software industry to understand perception of employees towards employers and take necessary steps to reduce the turnover intentions.
\end{abstract}

Keywords: Turnover Intentions, Software Industry, Employers

\section{INTRODUCTION}

Software Development Life Cycle is a systems approach to problem solving and is made up of several phases, each comprised of well define input and output milestones. Testing phase has much importance in Software Development Life Cycle due to a major contribution in finding and correcting the errors and it was performed by Software Testers. The field of Information Technology has faced a revolution in past decades and has opened new areas of success and opportunities for entrepreneur globally. But now the software industry is facing a serious problem of high turnover. High tumover will affect the project success. The roles of software testers have significant impact on the project success. The following problems will encounter when software testers left their current job during Testing Phase.

$>$ Functional Knowledge on the application.

$>$ Experience on the application

$>$ Experience on the usage of testing tools with respect to specific project

$>$ Client interactions

$>$ Collaboration within the testing team

$>$ Collaboration within the development team

$>$ Time to build a new member

\section{LITERATURE REVIEW}

Zhigang Wu [1] et al, analyze the impact of employee turnover along controllable variable and Uncontrollable variable. Zhang Guangyu [2] et al, developed a model to understand the talents loss risk using an evaluating indexes system with fuzzy comprehensive evaluation method. Xinhui Jiang [3] et al, while examining to study the turnover intentions with respect to impact and supervisors' monitoring, results indicate that former did not reduce turnover intentions, but when the later was high, turnover intentions was high. Dayong Wang [4], conducted a study to determine turnover intentions of new employees based on 6 types of pressure such as pressure from work itself, organizational management pressure, role ambiguity and role conflict pressure, interpersonal relationship and communication pressure, career development pressure and personal qualities pressure. Personal qualities pressure has no impact on turnover intentions. Zhang Jiandong [5], conducted a study in IT industry to determine employee engagement such as work input, organizational identity and work-happiness with respect to gender, age, marital status, education and working time. Qin Zhou [6], suggests that low perceived organization support and low job satisfaction leads to high turnover intentions. Dawn Owens [7] et al, conducted a study and classified into two categories to retain the talented employees such as Non-Monetary (Intrinsic) and Monetary (Extrinsic) characteristics. Jo Ellen Moore [8], results suggests that low affective commitment, high perceived job alternatives, and high psychological futility of voice are the primary determinants of turnover. Oksan Imamoglu [9] et al, determines the attributes for project success or failure. The causes of failure are went over time, went over budget, no organizational benefits and low user satisfaction. The reasons for project success are good project management and leadership, effective planning, executive and sponsor commitment, total organization and project team commitment. Janice M. Bum [10] et al identified five key dimensions for motivating their jobs in computing professionals such as skill variety, task identity, task significance, autonomy and feedback. F. Calisir [11] Y.P.S.Kanwar [13] Sinem Aydogdu [38] et al, determines that organizational commitment and job satisfaction predicts intention to quit the job. Sohrab Ahmad [29] et al, determined organizational commitment has significant impact on turnover intentions of call center personnel in Pakistan. Caramollah Daneshfard [37] et al, identifies that there is a direct relationship between organizational commitment and job satisfaction. Abdul Rahman [16] et al, determines perceived alternative job opportunities had significant impact on turnover intentions among IT professionals in Pakistan. Janice Anna Knights [22] et al, determines Psychological Contract Violation have significant impact on job satisfaction and organizational commitment. Jordan Shropshire[23] et al, identified that Stress, job insecurity and burnout are the major turnover intentions to leave the IT field. Age was not found to be any reasonable relationships.

\section{SCOPE OF THE STUDY}

Employee is one of the biggest asset for an organization. The organization has to understand the needs and keep them with 
safe and happy. In our present research work, we have exclusively taken new entrants in software testing professionals for the study and we have cross compared the various factors for their turnover intentions in Software Industry under present scenario.

In the early work done by researchers, Y.P.S.Kanwar[13] Sinem Aydogdu[38] Caramollah Daneshfard[37] Sohrab Ahmad[29] et al have taken two modules such as module 1 [Job satisfaction] and module 2[Organizational commitment] and they have applied correlation and/or regression analysis and obtain crucial results. Qin Zhou[6] Abdul Rahman[16] Jordan Shropshire[23] et al have taken three modules such as [Locus of control, Perceived Organizational support, Job Satisfaction], [Job Satisfaction, Organizational Commitment, Perceived Alternative Job Opportunities] and [Impact of Stress, Job Insecurity, Burnout] by using factor analysis [6], correlation analysis [16] and structural equation model [23] they obtain the results. Qin Zhou [6] Abdul Rahman[16] Jordan Shropshire[23] et al have taken three modules such as [Locus of control, Perceived Organizational support, Job Satisfaction], [Job Satisfaction, Organizational Commitment, Perceived Alternative Job Opportunities] and [Impact of Stress, Job Insecurity, Burnout] by applying factor analysis[6], correlation analysis[16] and structural equation model[23] they obtain the results. Calisir[11] et al have taken five modules such as the[Impact of stressors, Job stress, Organizational commitment, Locus of control and Job satisfaction], by applying multiple regression analysis they obtain the results. Previous research work is pertaining to IT professionals and Non-IT professionals in general and they have thoroughly tabulated the results.

Our research interest has taken from the above lead and we have selected four modules such as module 1[Organization satisfaction], module 2[Job satisfaction], module 3 [Interpersonal relationship with supervisor's] and module 4 [Life satisfaction]. We have applied correlation and regression analysis to compare these four modules and obtain the results which is shown in later pages. The unique work we can defend in our work is that the particular four combinations is not been travelled by many researchers and that to among software testing professionals.

\section{OBJECTIVES OF THE STUDY}

The study has the following objectives

$>$ To study the demographic profile of the respondents.

$>$ To understand the satisfaction level according to gender about the organization satisfaction, job satisfaction, interpersonal relationship with supervisors and life satisfaction.

$>$ To examine the turnover intentions in software testing professionals.

\section{HYPOTHESIS OF THE STUDY}

H1: There is no significant difference between gender and turnover intentions in software testing professionals at 5 percent level of significance.
H2: There is no significant difference between age and turnover intentions in software testing professionals at 5 percent level of significance.

H3: There is no significant difference between income level and turnover intentions in software testing professionals at 5 percent level of significance.

H4: Male employees will have greater organization satisfaction, job satisfaction, interpersonal relationship with supervisor's and life satisfaction and lesser turnover intent compared to Female employees.

H5: Organization Satisfaction is negatively correlated with turnover intent.

H6: Job Satisfaction is negatively correlated with turnover intent.

H7: Interpersonal relationship with supervisors is negatively correlated with turnover intent.

H8: Satisfaction in Life is negatively correlated with turnover intent.

\section{RESEARCH METHODOLOGY}

\begin{tabular}{|c|c|}
\hline Type of Research & $\begin{array}{l}\text { Descriptive cum Exploratory in } \\
\text { nature }\end{array}$ \\
\hline Nature of data & Primary and Secondary data \\
\hline Data Collection & $\begin{array}{l}\text { Questionnaire was prepared and } \\
\text { distributed to the respondents; } \\
\text { responses were collected through } \\
\text { personal interview, E-mails and } \\
\text { hosted web site }\end{array}$ \\
\hline Area of the study & Chennai \\
\hline Population frame & $\begin{array}{l}\text { Employees' from Software } \\
\text { Industry }\end{array}$ \\
\hline Sampling Unit & $\begin{array}{l}\text { Employees' from Software Testing } \\
\text { Professionals }\end{array}$ \\
\hline Sample size & 135 \\
\hline Tools for analysis & $\begin{array}{l}\text { Descriptive Statistics: } \\
\text { Frequency, Cross Table, ANOVA, } \\
\text { Correlation and Regression }\end{array}$ \\
\hline $\begin{array}{l}\text { Questionnaire } \\
\text { Analysis[Reliability] }\end{array}$ & $\begin{array}{l}\text { Cronbach Alpha: } \\
\text { Organization Satisfaction }=8 \\
\text { items, } 0.956 \text {, Job Satisfaction }=13 \\
\text { items, } 0.773 \text {, Interpersonal } \\
\text { relationship with supervisor's }=9 \\
\text { items, } 0.976 \text {, Life Satisfaction }=5 \\
\text { items, } 0.663 \text {, Turnover Intentions }= \\
2 \text { items, } 0.933\end{array}$ \\
\hline Scaling Method & $\begin{array}{l}\text { Likert Scale: Strong Agree }=5, \\
\text { Agree }=4, \text { Neutral }=3, \text { Disagree }= \\
2, \text { Strong Disagree }=1\end{array}$ \\
\hline
\end{tabular}

\section{RESULTS}

Various statistical analyses were conducted to test the different hypothesis. The results of the study are presented in four sub-sections. The first section shows the demographic 
profile of the respondents. The section shows the ANOVA results for significant different in hypothesis. The third section describes correlation results and fourth section presents regression analysis.

\subsection{Demographic Profile of the Respondents} Table 1: Demographic characteristics

\begin{tabular}{|l|l|c|}
\hline \multicolumn{1}{|c|}{ Parameters } & \multicolumn{1}{|c|}{ Category } & $\begin{array}{c}\text { Number of } \\
\text { Respondents }\end{array}$ \\
\hline Gender & Male & 67 \\
& Female & 68 \\
\hline Age & $<25$ & 122 \\
& 26 to 30 & 13 \\
\hline Marital Status & Married & 13 \\
& Unmarried & 122 \\
\hline Education & UG & 55 \\
Qualification & PG & 31 \\
& Professional & 49 \\
\hline Annual income in & $<=3,00,000$ & 55 \\
Rs. & $3,00,001$ to $=$ & 80 \\
& $6,00,000$ & 71 \\
\hline Domain & Retail Industry & 64 \\
\hline Process Model & Health Care & 75 \\
\hline Working hours & Waterfall & 60 \\
\hline Per week & Spiral & 60 \\
\hline Source: Primary data & 40 to 50 & \\
\hline & & \\
\hline
\end{tabular}

Source: Primary data

\subsection{ANOVA Results}

The Table 2, 3 and 4 shows that there is a significant difference exists among Gender, Education and Income level with respect to turnover intentions. Hence $\mathrm{H} 1, \mathrm{H} 2$ and $\mathrm{H} 3$ are rejected. Table 5 shows that comparative study of male employees and female employees on different group variables such as organization satisfaction, job satisfaction, interpersonal relationship with supervisor's, life satisfaction and turnover intentions. The result reveals that male employees are more satisfied in different group variables and lesser turnover intent. Hence $\mathrm{H} 4$ is accepted.

Table 2: ANOVA

\begin{tabular}{|l|r|r|r|r|r|}
\hline & $\begin{array}{c}\text { Sum of } \\
\text { Squares }\end{array}$ & Df & $\begin{array}{c}\text { Mean } \\
\text { Square }\end{array}$ & F & Sig. \\
\hline $\begin{array}{l}\text { Between } \\
\text { Groups }\end{array}$ & 23.713 & 1 & 23.713 & 12.8 & .001 \\
\hline $\begin{array}{l}\text { Within } \\
\text { Groups }\end{array}$ & 245.221 & 133 & 1.844 & & \\
\hline Total & 268.933 & 134 & & & \\
\hline
\end{tabular}

Source: Primary data

Table 3: ANOVA

\begin{tabular}{|l|r|r|r|r|r|}
\hline & $\begin{array}{r}\text { Sum of } \\
\text { Squares }\end{array}$ & df & $\begin{array}{c}\text { Mean } \\
\text { Square }\end{array}$ & F & Sig. \\
\hline $\begin{array}{l}\text { Between } \\
\text { Groups }\end{array}$ & 13.449 & 2 & 6.724 & 3.47 & .034 \\
\hline $\begin{array}{l}\text { Within } \\
\text { Groups }\end{array}$ & 255.485 & 132 & 1.935 & & \\
\hline Total & 268.933 & 134 & & & \\
\hline
\end{tabular}

Source: Primary data
Table 4: ANOVA

\begin{tabular}{|l|c|c|c|c|c|}
\hline & $\begin{array}{c}\text { Sum of } \\
\text { Squares }\end{array}$ & df & $\begin{array}{c}\text { Mean } \\
\text { Square }\end{array}$ & F & Sig. \\
\hline $\begin{array}{l}\text { Between } \\
\text { Groups }\end{array}$ & 13.246 & 1 & 13.246 & 6.89 & .010 \\
\hline $\begin{array}{l}\text { Within } \\
\text { Groups }\end{array}$ & 255.688 & 133 & 1.922 & & \\
\hline Total & 268.933 & 134 & & & \\
\hline
\end{tabular}

Source: Primary data

Table 5: Comparison of Male and Female Groups on Different variables [ $\mathbf{N}=135]$

\begin{tabular}{|l|lc|lc|}
\hline \multicolumn{5}{|c|}{ Sex } \\
\hline Variables & \multicolumn{2}{|c|}{ Male ( N = 67) } & \multicolumn{2}{l|}{ Female ( N = } \\
& Mean & S.D & 68) \\
Mean & S.D \\
\hline $\begin{array}{l}\text { Organization } \\
\text { Satisfaction }\end{array}$ & 3.287 & 0.941 & 2.572 & 0.91 \\
\hline Job Satisfaction & 3.691 & 0.482 & 3.37 & 0.408 \\
\hline $\begin{array}{l}\text { Interpersonal } \\
\text { Relationship with } \\
\text { Supervisor's }\end{array}$ & 3.249 & 0.983 & 2.428 & 0.87 \\
\hline Life Satisfaction & 3.397 & 0.544 & 3.015 & 0.616 \\
\hline $\begin{array}{l}\text { Turnover } \\
\text { Intentions }\end{array}$ & 3.00 & 1.348 & 3.838 & 1.367 \\
\hline
\end{tabular}

Source: Primary data

Table 6: Opinion about Organization Satisfaction

\begin{tabular}{|c|c|c|c|c|c|}
\hline & SA & A & $\mathrm{N}$ & D & SD \\
\hline $\begin{array}{l}\text { I am familiar } \\
\text { with } \\
\text { organization's } \\
\text { current vision \& } \\
\text { mission }\end{array}$ & $\begin{array}{c}49 \\
(36.3)\end{array}$ & $\begin{array}{c}69 \\
(51.1)\end{array}$ & $\begin{array}{c}12 \\
(8.9)\end{array}$ & $\begin{array}{c}4 \\
(3.0)\end{array}$ & $\begin{array}{c}1 \\
(.7 \\
)\end{array}$ \\
\hline $\begin{array}{l}\text { The Organization } \\
\text { is maintaining } \\
\text { consistent } \\
\text { policies }\end{array}$ & $\begin{array}{c}32 \\
(23.7)\end{array}$ & $\begin{array}{c}21 \\
(15.6)\end{array}$ & $\begin{array}{c}2 \\
(1.5)\end{array}$ & $\begin{array}{c}23 \\
(17.0)\end{array}$ & $\begin{array}{c}57 \\
(4 \\
2.2 \\
)\end{array}$ \\
\hline $\begin{array}{l}\text { The Organization } \\
\text { motivates } \\
\text { employees }\end{array}$ & $\begin{array}{c}6 \\
(4.4)\end{array}$ & $\begin{array}{c}49 \\
(36.3)\end{array}$ & $\begin{array}{c}7 \\
(5.2)\end{array}$ & $\begin{array}{c}30 \\
(22.2)\end{array}$ & $\begin{array}{c}43 \\
(3 \\
1.9 \\
)\end{array}$ \\
\hline $\begin{array}{l}\text { The Organization } \\
\text { openly discusses } \\
\text { job related issues } \\
\text { with the } \\
\text { employees }\end{array}$ & $\begin{array}{c}6 \\
(4.4)\end{array}$ & $\begin{array}{c}30 \\
(22.2)\end{array}$ & $\begin{array}{c}40 \\
(29.6 \\
)\end{array}$ & $\begin{array}{c}51 \\
(37.8)\end{array}$ & $\begin{array}{r}8 \\
(5 . \\
9)\end{array}$ \\
\hline $\begin{array}{l}\text { The Organization } \\
\text { follows standard } \\
\text { appraisal } \\
\text { procedure }\end{array}$ & $\begin{array}{c}4 \\
(3.0)\end{array}$ & $\begin{array}{c}54 \\
(40.0)\end{array}$ & $\begin{array}{c}13 \\
(9.6)\end{array}$ & $\begin{array}{c}11 \\
(8.1)\end{array}$ & $\begin{array}{c}53 \\
(3 \\
9.3 \\
)\end{array}$ \\
\hline $\begin{array}{l}\text { The Organization } \\
\text { gives scope for } \\
\text { career } \\
\text { advancements }\end{array}$ & $\begin{array}{c}6 \\
(4.4)\end{array}$ & $\begin{array}{c}31 \\
(23.0)\end{array}$ & $\begin{array}{c}23 \\
(17.0 \\
)\end{array}$ & $\begin{array}{c}75 \\
(55.6)\end{array}$ & 0 \\
\hline $\begin{array}{l}\text { The Organization } \\
\text { takes pride in my } \\
\text { accomplishments } \\
\text { at work }\end{array}$ & $\begin{array}{c}1 \\
(.7)\end{array}$ & $\begin{array}{c}55 \\
(40.7)\end{array}$ & $\begin{array}{c}61 \\
(45.2 \\
)\end{array}$ & $\begin{array}{c}10 \\
(7.4)\end{array}$ & $\begin{array}{l}08 \\
(5 . \\
9)\end{array}$ \\
\hline $\begin{array}{l}\text { The Organization } \\
\text { is open for } \\
\text { feedback }\end{array}$ & $\begin{array}{c}5 \\
(3.7)\end{array}$ & $\begin{array}{c}33 \\
(24.4)\end{array}$ & $\begin{array}{c}41 \\
(30.4 \\
)\end{array}$ & $\begin{array}{c}17 \\
(12.6)\end{array}$ & $\begin{array}{c}39 \\
(2 \\
8.9 \\
)\end{array}$ \\
\hline
\end{tabular}


Source: Primary data [SA=Strongly Agree, A=Agree, $\mathrm{N}=$ Neutral, $\mathrm{D}=$ Disagree, $\mathrm{SD}=$ Strongly Disagree]

Table 7: Opinion about Job Satisfaction

\begin{tabular}{|c|c|c|c|c|c|}
\hline & SA & $\mathrm{A}$ & $\mathrm{N}$ & $\mathrm{D}$ & SD \\
\hline $\begin{array}{l}\text { I am fairly paid } \\
\text { for the job i } \\
\text { perform }\end{array}$ & $\begin{array}{c}55 \\
(40.7)\end{array}$ & $\begin{array}{c}49 \\
(36.3)\end{array}$ & $\begin{array}{c}11 \\
(8.1)\end{array}$ & $\begin{array}{c}13 \\
(9.6)\end{array}$ & $\begin{array}{c}08 \\
(5.2)\end{array}$ \\
\hline $\begin{array}{l}\text { I have sufficient } \\
\text { resources to do } \\
\text { my job }\end{array}$ & $\begin{array}{c}75 \\
(55.6)\end{array}$ & $\begin{array}{c}40 \\
(29.6)\end{array}$ & $\begin{array}{c}11 \\
(8.1)\end{array}$ & $\begin{array}{c}05 \\
(3.7)\end{array}$ & $\begin{array}{c}04 \\
(3.0)\end{array}$ \\
\hline $\begin{array}{l}\text { I am relatively } \\
\text { free to my job }\end{array}$ & $\begin{array}{c}55 \\
(40.7)\end{array}$ & $\begin{array}{c}62 \\
(45.9)\end{array}$ & $\begin{array}{c}11 \\
(8.1)\end{array}$ & $\begin{array}{c}04 \\
(3.0)\end{array}$ & $\begin{array}{c}03 \\
(2.2)\end{array}$ \\
\hline $\begin{array}{l}\text { I am having good } \\
\text { relationship with } \\
\text { my team members }\end{array}$ & $\begin{array}{c}17 \\
(12.6)\end{array}$ & $\begin{array}{c}62 \\
(45.9)\end{array}$ & $\begin{array}{c}14 \\
(10.4)\end{array}$ & $\begin{array}{c}38 \\
(28.1)\end{array}$ & $\begin{array}{c}04 \\
(3.0)\end{array}$ \\
\hline $\begin{array}{l}\text { I am satisfied with } \\
\text { my supervisor(s) } \\
\text { role }\end{array}$ & $\begin{array}{c}06 \\
(4.4)\end{array}$ & $\begin{array}{c}44 \\
(32.6)\end{array}$ & $\begin{array}{c}33 \\
(24.4)\end{array}$ & $\begin{array}{c}16 \\
(11.9)\end{array}$ & $\begin{array}{c}36 \\
(26 . \\
7) \\
\end{array}$ \\
\hline & $\begin{array}{c}11 \\
(8.1)\end{array}$ & $\begin{array}{c}110 \\
(81.5)\end{array}$ & $\begin{array}{c}11 \\
(8.1)\end{array}$ & $\begin{array}{c}02 \\
(1.5)\end{array}$ & $\begin{array}{c}01 \\
(.7)\end{array}$ \\
\hline $\begin{array}{l}\text { My present job } \\
\text { demands frequent } \\
\text { extended working } \\
\text { hours }\end{array}$ & $\begin{array}{c}10 \\
(7.4)\end{array}$ & $\begin{array}{c}63 \\
(46.7)\end{array}$ & $\begin{array}{c}18 \\
(13.3)\end{array}$ & $\begin{array}{c}20 \\
(14.8)\end{array}$ & $\begin{array}{c}24 \\
(17 . \\
8)\end{array}$ \\
\hline $\begin{array}{l}\text { I feel I have a } \\
\text { secured job }\end{array}$ & $\begin{array}{c}73 \\
(54.1) \\
\end{array}$ & $\begin{array}{c}49 \\
(36.3)\end{array}$ & $\begin{array}{c}13 \\
(9.6)\end{array}$ & 0 & 0 \\
\hline $\begin{array}{l}\text { There is a Stress } \\
\text { in my job }\end{array}$ & $\begin{array}{c}07 \\
(5.2) \\
\end{array}$ & $\begin{array}{c}34 \\
(25.2) \\
\end{array}$ & $\begin{array}{c}13 \\
(9.6) \\
\end{array}$ & $\begin{array}{c}71 \\
(52.6) \\
\end{array}$ & $\begin{array}{c}10 \\
(7.4) \\
\end{array}$ \\
\hline $\begin{array}{l}\text { I am encouraged } \\
\text { to come up with } \\
\text { innovative ideas } \\
\text { on the job }\end{array}$ & $\begin{array}{c}22 \\
(16.3)\end{array}$ & $\begin{array}{c}35 \\
(25.9)\end{array}$ & $\begin{array}{c}58 \\
(43.0)\end{array}$ & $\begin{array}{c}12 \\
(8.9)\end{array}$ & $\begin{array}{c}08 \\
(5.9)\end{array}$ \\
\hline $\begin{array}{l}\text { There is a } \\
\text { recognition in my } \\
\text { job }\end{array}$ & $\begin{array}{c}19 \\
(14.1)\end{array}$ & $\begin{array}{c}44 \\
(32.6)\end{array}$ & $\begin{array}{c}16 \\
(11.9)\end{array}$ & $\begin{array}{c}47 \\
(34.8)\end{array}$ & $\begin{array}{c}09 \\
(6.7)\end{array}$ \\
\hline $\begin{array}{l}\text { I am aware of } \\
\text { promotional } \\
\text { opportunities and } \\
\text { also i have a } \\
\text { chance of } \\
\text { advancement }\end{array}$ & $\begin{array}{c}21 \\
(15.6)\end{array}$ & $\begin{array}{c}42 \\
(31.1)\end{array}$ & $\begin{array}{c}57 \\
(42.2)\end{array}$ & $\begin{array}{c}09 \\
(6.7)\end{array}$ & $\begin{array}{c}06 \\
(4.4)\end{array}$ \\
\hline $\begin{array}{l}\text { The way } \\
\text { organizational } \\
\text { policies are put } \\
\text { into practice is } \\
\text { good }\end{array}$ & $\begin{array}{c}19 \\
(14.1)\end{array}$ & $\begin{array}{c}30 \\
(22.2)\end{array}$ & $\begin{array}{c}48 \\
(35.6)\end{array}$ & $\begin{array}{c}22 \\
(16.3)\end{array}$ & $\begin{array}{c}16 \\
(11 . \\
9)\end{array}$ \\
\hline
\end{tabular}

Source: Primary data [SA=Strongly Agree, $\mathrm{A}=$ Agree,

$\mathrm{N}=$ Neutral, $\mathrm{D}=$ Disagree, $\mathrm{SD}=$ Strongly Disagree]

Table 8: Opinion about Interpersonal Relationship with Supervisor's

\begin{tabular}{|l|c|c|c|c|c|}
\hline & SA & A & N & D & SD \\
\hline $\begin{array}{l}\text { There is a smooth } \\
\text { interpersonal } \\
\text { communication }\end{array}$ & $\begin{array}{c}44 \\
(32.6)\end{array}$ & $\begin{array}{c}26 \\
(19.3)\end{array}$ & $\begin{array}{c}21 \\
(15.6)\end{array}$ & $\begin{array}{c}44 \\
(32.6)\end{array}$ & 0 \\
\hline $\begin{array}{l}\text { I get sufficient } \\
\text { knowledge from } \\
\text { my supervisor(s) }\end{array}$ & $\begin{array}{c}32 \\
(23.0)\end{array}$ & $\begin{array}{c}24 \\
(17.8)\end{array}$ & $\begin{array}{c}25 \\
(18.5)\end{array}$ & $\begin{array}{c}47 \\
(34.8)\end{array}$ & $\begin{array}{c}08 \\
(5.9)\end{array}$ \\
\hline $\begin{array}{l}\text { My supervisor(s) } \\
\text { is consistent in } \\
\text { applying the rules } \\
\text { to all the }\end{array}$ & $\begin{array}{c}02 \\
(1.5)\end{array}$ & $\begin{array}{c}27 \\
(20.0)\end{array}$ & $\begin{array}{c}26 \\
(19.3)\end{array}$ & $\begin{array}{c}27 \\
(20.0)\end{array}$ & $\begin{array}{c}53 \\
(39.3)\end{array}$ \\
\hline
\end{tabular}

\begin{tabular}{|c|c|c|c|c|c|}
\hline employees & & & & & \\
\hline $\begin{array}{l}\text { My supervisor(s) } \\
\text { treats me with } \\
\text { respect }\end{array}$ & $\begin{array}{c}01 \\
(.7)\end{array}$ & $\begin{array}{c}68 \\
(50.4)\end{array}$ & $\begin{array}{c}11 \\
(8.1)\end{array}$ & $\begin{array}{c}55 \\
(40.7)\end{array}$ & 0 \\
\hline $\begin{array}{l}\text { My supervisor(s) } \\
\text { gives me a } \\
\text { reasonable work }\end{array}$ & $\begin{array}{c}03 \\
(2.2)\end{array}$ & $\begin{array}{c}54 \\
(40.0)\end{array}$ & $\begin{array}{c}63 \\
(46.7)\end{array}$ & $\begin{array}{c}12 \\
(8.9)\end{array}$ & $\begin{array}{c}03 \\
(2.2)\end{array}$ \\
\hline $\begin{array}{l}\text { My supervisor(s) } \\
\text { accepts my ideas } \\
\text { if it is valid }\end{array}$ & $\begin{array}{c}01 \\
(.7)\end{array}$ & $\begin{array}{c}52 \\
(38.5)\end{array}$ & $\begin{array}{c}77 \\
(57.0)\end{array}$ & $\begin{array}{c}04 \\
(3.0)\end{array}$ & $\begin{array}{c}01 \\
(.7)\end{array}$ \\
\hline $\begin{array}{l}\text { My supervisor(s) } \\
\text { provides } \\
\text { performance } \\
\text { feedback(ie timely } \\
\& \text { consistent } \\
\text { basis) }\end{array}$ & $\begin{array}{c}02 \\
(1.5)\end{array}$ & $\begin{array}{c}53 \\
(39.3)\end{array}$ & $\begin{array}{c}02 \\
(1.5)\end{array}$ & $\begin{array}{c}17 \\
(12.6)\end{array}$ & $\begin{array}{c}61 \\
(45.2)\end{array}$ \\
\hline $\begin{array}{l}\text { My supervisor(s) } \\
\text { provides me with } \\
\text { cross training } \\
\text { outside of my } \\
\text { specialization }\end{array}$ & $\begin{array}{c}03 \\
(2.2)\end{array}$ & $\begin{array}{c}31 \\
(23.0)\end{array}$ & $\begin{array}{c}22 \\
(16.3)\end{array}$ & $\begin{array}{c}20 \\
(14.8)\end{array}$ & $\begin{array}{c}59 \\
(43.7)\end{array}$ \\
\hline $\begin{array}{l}\text { My supervisor(s) } \\
\text { provides me with } \\
\text { opportunities for } \\
\text { professional } \\
\text { growth }\end{array}$ & $\begin{array}{c}02 \\
(1.5)\end{array}$ & $\begin{array}{c}29 \\
(21.5)\end{array}$ & $\begin{array}{c}22 \\
(16.3)\end{array}$ & $\begin{array}{c}18 \\
(13.3)\end{array}$ & $\begin{array}{c}64 \\
(47.4)\end{array}$ \\
\hline
\end{tabular}

Source: Primary data [SA=Strongly Agree, $\mathrm{A}=$ Agree, $\mathrm{N}=$ Neutral, $\mathrm{D}=$ Disagree, $\mathrm{SD}=$ Strongly Disagree]

Table 9: Opinion about Life Satisfaction

\begin{tabular}{|c|c|c|c|c|c|}
\hline & SA & $\mathrm{A}$ & $\mathrm{N}$ & $\mathrm{D}$ & $\mathrm{SD}$ \\
\hline $\begin{array}{c}\text { In most ways my } \\
\text { life is close to my } \\
\text { ambition \& wishes }\end{array}$ & $\begin{array}{c}25 \\
(18.5)\end{array}$ & $\begin{array}{c}65 \\
(48.1)\end{array}$ & $\begin{array}{c}18 \\
(13.3)\end{array}$ & $\begin{array}{c}19 \\
(14.1)\end{array}$ & $\begin{array}{c}08 \\
(5.9)\end{array}$ \\
\hline $\begin{array}{c}\text { The conditions of } \\
\text { my life are excellent }\end{array}$ & $\begin{array}{c}11 \\
(8.1)\end{array}$ & $\begin{array}{c}51 \\
(37.8)\end{array}$ & $\begin{array}{c}43 \\
(31.9)\end{array}$ & $\begin{array}{c}19 \\
(14.1)\end{array}$ & $\begin{array}{c}11 \\
(8.1)\end{array}$ \\
\hline $\begin{array}{c}\text { I am satisfied with } \\
\text { my life }\end{array}$ & $\begin{array}{c}15 \\
(11.1)\end{array}$ & $\begin{array}{c}30 \\
(22.2)\end{array}$ & $\begin{array}{c}61 \\
(45.2)\end{array}$ & $\begin{array}{c}11 \\
(8.1)\end{array}$ & $\begin{array}{c}18 \\
(13.3)\end{array}$ \\
\hline $\begin{array}{c}\text { So far i have got the } \\
\text { important things i } \\
\text { wanted in life }\end{array}$ & $\begin{array}{c}03 \\
(2.2)\end{array}$ & $\begin{array}{c}39 \\
(28.9)\end{array}$ & $\begin{array}{c}69 \\
(51.1)\end{array}$ & $\begin{array}{c}21 \\
(15.6)\end{array}$ & $\begin{array}{c}03 \\
(2.2)\end{array}$ \\
\hline $\begin{array}{c}\text { I would change } \\
\text { almost nothing } \\
\text { hereafter in my life }\end{array}$ & $\begin{array}{c}05 \\
(3.7)\end{array}$ & $\begin{array}{c}10 \\
(7.4)\end{array}$ & $\begin{array}{c}102 \\
(75.6)\end{array}$ & $\begin{array}{c}11 \\
(8.1)\end{array}$ & $\begin{array}{c}07 \\
(5.2)\end{array}$ \\
\hline
\end{tabular}

Source: Primary data [SA=Strongly Agree, A=Agree, $\mathrm{N}=$ Neutral, $\mathrm{D}=$ Disagree, $\mathrm{SD}=$ Strongly Disagree]

\subsection{Correlation Results}

From table 10 the result reveals that there is a strong negative relationship exists between organizational satisfaction, job satisfaction and interpersonal relationship with supervisors with respect to turnover intentions. A moderate negative relationship exists between life satisfaction and turnover intentions. Thus decrease in organizational satisfaction, job satisfaction, and interpersonal relationship with supervisor's and life satisfaction will increase turnover intentions. Thus Hypothesis H5, H6, H7 and $\mathrm{H} 8$ are accepted. 
Table 10 : Correlations between Different Variables $(\mathrm{N}=135)$

\begin{tabular}{|c|c|c|c|c|c|c|}
\hline & & $\begin{array}{l}\text { Organizatio } \\
\mathrm{n} \\
\text { Satisfaction }\end{array}$ & $\begin{array}{c}\text { Job } \\
\text { Satisfaction }\end{array}$ & $\begin{array}{l}\text { Interpersonal } \\
\text { Relationship } \\
\text { with } \\
\text { Supervisor's }\end{array}$ & $\begin{array}{c}\text { Life } \\
\text { Satisfaction }\end{array}$ & $\begin{array}{l}\text { Turnover } \\
\text { Intentions }\end{array}$ \\
\hline \multirow{2}{*}{$\begin{array}{l}\text { Organization } \\
\text { Satisfaction }\end{array}$} & Pearson Correlation & 1 & $.725^{* *}$ & $.950^{* * *}$ & $.468^{* * *}$ & $-.876^{* * *}$ \\
\hline & Sig. (2-tailed) & & .000 & .000 & .000 & .000 \\
\hline \multirow[t]{2}{*}{ Job Satisfaction } & Pearson Correlation & $.725^{* * *}$ & 1 & $.766^{* * *}$ & $.436^{* *}$ & $-.726^{* * 6}$ \\
\hline & Sig. (2-tailed) & .000 & & .000 & .000 & .000 \\
\hline \multirow{2}{*}{$\begin{array}{l}\text { Interpersonal } \\
\text { Relationship }\end{array}$} & Pearson Correlation & $.950^{* *}$ & $.766^{* *}$ & 1 & $.504^{* *}$ & $-.898^{* * *}$ \\
\hline & Sig. (2-tailed) & .000 & .000 & & .000 & .000 \\
\hline \multirow[t]{2}{*}{ Life Satisfaction } & Pearson Correlation & $.468^{* *}$ & $.436^{* *}$ & $.504^{* * *}$ & 1 & $-.422^{* * *}$ \\
\hline & Sig. (2-tailed) & .000 & .000 & .000 & & .000 \\
\hline \multirow{2}{*}{$\begin{array}{l}\text { Turnover } \\
\text { Intentions }\end{array}$} & Pearson Correlation & $-.876^{* *}$ & $-.726^{* *}$ & $-.898^{* * *}$ & $-.422^{* *}$ & 1 \\
\hline & Sig. (2-tailed) & .000 & .000 & .000 & .000 & \\
\hline
\end{tabular}

Source: Primary data

\subsection{Regression Results}

Based on the results of the correlation analysis, stepwise regression analysis were carried out to determine true relationship exists between four independent variables such as organization satisfaction, job satisfaction, interpersonal relationship with supervisor's, life satisfaction and one dependent variable is turnover intentions. The table 12 reveals that it is significant for predicting independent variables and dependent variable. The table 13 reveals that Organization Satisfaction and Interpersonal relationship with supervisors are the strong predictors for turnover intentions among new entrants in software testing professionals.

Table 11: Model Summary

\begin{tabular}{|l|c|r|r|r|}
\hline Model & $\mathrm{R}$ & $\begin{array}{c}\mathrm{R} \\
\text { Square }\end{array}$ & $\begin{array}{c}\text { Adjusted R } \\
\text { Square }\end{array}$ & $\begin{array}{c}\text { Std. Error of } \\
\text { the Estimate }\end{array}$ \\
\hline 1 & $.904^{\mathrm{a}}$ & .817 & .811 & .61596 \\
\hline
\end{tabular}

Source: Primary data

a. Predictors: (Constant), Life Satisfaction, Job, Organization, Interpersonal Relationship

Table 12: ANOVA

\begin{tabular}{|l|r|r|r|r|r|}
\hline Model & $\begin{array}{c}\text { Sum of } \\
\text { Squares }\end{array}$ & Df & $\begin{array}{c}\text { Mean } \\
\text { Square }\end{array}$ & F & Sig. \\
\hline $\begin{array}{l}\text { Regressi } \\
\text { on }\end{array}$ & 219.611 & 4 & 54.903 & 144.709 & $\begin{array}{r}.000 \\
\text { a }\end{array}$ \\
\hline Residual & 49.322 & 130 & .379 & & \\
\hline Total & 268.933 & 134 & & & \\
\hline
\end{tabular}

Source: Primary data

a.Predictors: (Constant), Life Satisfaction, Job, Organization, Interpersonal Relationship

b.Dependent Variable: Turnover Intentions
Table 13: Coefficients

\begin{tabular}{|l|r|r|r|c|c|}
\hline Model & \multicolumn{2}{|c|}{$\begin{array}{l}\text { Unstandardize } \\
\text { d Coefficients }\end{array}$} & $\begin{array}{c}\text { Standar } \\
\text { dized } \\
\text { Coeffic } \\
\text { ients }\end{array}$ & T & Sig. \\
\cline { 2 - 5 } & $\mathrm{B}$ & $\begin{array}{c}\text { Std. } \\
\text { Error }\end{array}$ & Beta & & \\
\hline (Constant) & $\begin{array}{r}\mid c 59 \\
4\end{array}$ & .512 & & 14.824 & .000 \\
\hline $\begin{array}{l}\text { Organization } \\
\text { Satisfaction }\end{array}$ & -.343 & .172 & -.240 & -1.996 & .048 \\
\hline Job Satisfaction & -.297 & .176 & -.099 & -1.689 & .094 \\
\hline $\begin{array}{l}\text { Interpersonal } \\
\text { Relationship } \\
\text { with }\end{array}$ & -.862 & .183 & -.616 & -4.712 & .000 \\
Supervisor's & & & & & \\
\hline $\begin{array}{l}\text { Life } \\
\text { Satisfaction }\end{array}$ & .102 & .101 & .044 & 1.009 & .315 \\
\hline
\end{tabular}

Source: Primary data

a. Dependent Variable: Turnover Intentions

Figure 1

Organization Satisfaction

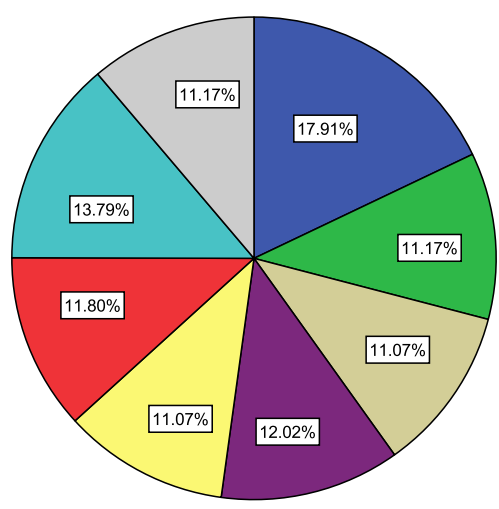

I amfamiliar with organization's
current vision \& mission The Organization is
maintaining maintaining
consistent policies $\square$ The Organization motivates employees
The Organization related issues with the employees The Organization appraisal procedure The Organization gives scope for career
advancements The Organization
takes pride in my $\square$ accomplishments at $\square$ The Organization is

Source: Primary Data

Figure 2 
Interpersonal Relationship with Supervisor's
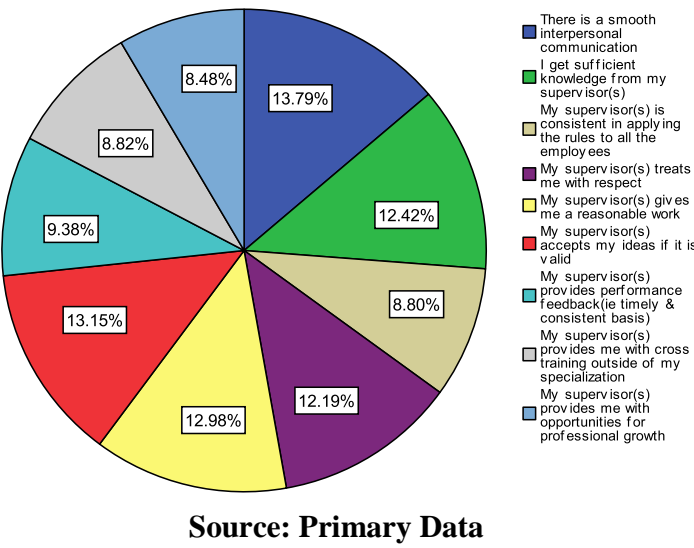

\section{CONCLUSION}

The study shows Organization Satisfaction and Interpersonal relationship with supervisors are the strong predictors for turnover intentions. Job Satisfaction and Life Satisfaction have no impact on turnover intentions among software testing professionals. Results from the Figure 1 and Figure 2, clearly indicates that maximum impact reason for turnover intentions among software testing professionals are lesser amount of motivation towards employees[11.07\%], No standard appraisal procedures for employee career benefits[11.07\%] and Supervisor's doesnot providing opportunities for professional growth[8.48\%]. From the table 5, it is understood that male employees are more satisfied when compared to female employees. It is also observed that male $=80.59 \%$ and female $=80.88 \%$ agrees that they are interested in doing testing job. The study can be extended to compare and contrast with a variety of other sectors. The study concludes that employee is the biggest asset for the organization. The organization has to revise the current policies for the new entrants and needs to motivate them. As well as the supervisors has to be more open mind in providing consistent performance feedback and give more opportunity for the new entrants to learn new things in the current technology in order to retain the talented young employees.

\section{ACKNOWLEDGEMENT}

I sincerely thank Mr.V.Balu, System Administrator, SCSVMV University for his kind co-operation.

\section{REFERENCES}

[1] Zhigang Wu \& Xiangmin Li, "Strategic Analysis of Employee Turnover”, IEEE, 978-1-4244-6581-1, 2011.

[2] Zhang Guangyu, Li Huajun \& Zhang Depeng, "Risk Management on Knowledge Employees Turnover in High-tech Firms -Based on Fuzzy Comprehensive Evaluation”, IEEE, 978-0-7695-3435-0/08, pp.399-402.

[3] Xinhui Jiang \& Qiang Zhang, "When Will Employees with High Impact Quit? The Interactive Effect of Impact and Supervisor's Monitoring on Chinese Employees'Turnover Intention", CROWN, 978-1-42446581-1, 2011.
[4] Dayong Wang ,"Research on the Influence of Working Pressure on Turnover Intention of New Employees", IEEE, 978-1-4244-6581-1, 2011.

[5] Zhang Jiandong, "Employee Engagement Investigation in IT Industry", IEEE, 978-1-4244-4507-3, 2009.

[6] Qin Zhou, "The Impact of Job Satisfaction Affect on Turnover Intention: An Empirical Study Based on the Circumstances of China", IEEE, 978-0-7695-3936-2, 2009, pp.220-223.

[7] Dawn Owens \& Deepak Khazanchi, "Best Practices for Retaining Global IT Talent”, IEEE, 1530-1605,2011, pp.1-12.

[8] Jo Ellen Moore, "Illuminating the Other Road: The Role of Voice in IT Turnover", IEEE, 1530-1605, 2011.

[9] Oksan Imamoglu \& Sitki Gozlu, "The Sources of Success and Failure of Information Technology Projects: Project Managers' Perspective", PICMET Proceedings, 27-31 July 2008, Cape Town, South Africa, pp.14301435 .

[10] Janice M Bum, Dr. D Couger, Louis Ma \& Dr. Hiram Tompkins, "Motivating IT Professionals - The Hong Kong Challenge", IEEE, 0073-11 29,1991, pp.524-529.

[11] F. Calisir, C.A. Gumussoy \& I. Iskin, "Factors Affecting Intention to Quit among IT Professionals", IEEE, 978-14244-4870-8, 2009, pp.49-53.

[12] J. Sheard \& S. Markham, "Creating an Interest in IT: A Gender Study", Proceedings of the International Conference on Computers in Education (ICCE'02), 07695-1509-6, 2002.

[13] Y.P.S. Kanwar, A.K.Singh \& Amitabh Deo Kodwani, “A Study of Job Satisfaction and Organizational Commitment and Turnover Intent among the IT and ITES Sector Employees", SAGE, DOI:10.1177/097226291201600103, 16(1), 2012, pp.2735 .

[14] Steven G. westlund \& John C. Hannon, "Retaining talent: Assessing job satisfaction facets most significantly related to software developer turnover intentions", Journal of Information Technology Management, ISSN: 1042-1319, Volume XIX, Number 4, 2008, pp.1-15.

[15] Prodromos D. Chatzoglou, Eftichia Vraimaki, Eleni Komsiou, Elena Polychrou \& Anastastios D. Diamantidis,Factors Affecting Accountants' Job Satisfaction and Turnover Intentions: A Structural Equation Model, $8^{\text {th }}$ International Conference on Enterprise Systems, Accounting and Logistics (8th ICESAL 2011) 11-12, July 2011, Thassos Island, Greece, pp.130-147.

[16] Abdul Rahman, S. M. M. Raza Naqvi \& M. Ismail Ramay,"Measuring Turnover Intention: A Study of IT Professionals in Pakistan", International Review of Business Research Papers, Vol. 4 No.3, June 2008, pp.45-55.

[17] Dr. Ify Dial \& Dr. Rao Nemani," Job Satisfaction: Key Factors Influencing Information Technology (IT) Professionals in Washington DC", IJCTA, ISSN:22296093, Vol 2 (4), pp.827-838. 
[18] Ify Diala, "Job Satisfaction Management System Approach on Information Technology Professionals", World Applied Programming, ISSN: 2222-2510, Vol (1), No (4), October 2011, pp.232-236.

[19] Kalim Ullah Khan, Syed Umar Farooq \& Muahmmad Imran Ullah,"The Relationship between Rewards and Employee Motivation in Commercial Banks of Pakistan", Research Journal of International Studies Issue 14,May 2010, pp.37-52.

[20] Mark E. McMurtrey, James P. Downey, Steven M. Zeltmann, \& William H. Friedman,"Critical Skill Sets of Entry-Level IT Professionals: An Empirical Examination of Perceptions from Field Personnel", Journal of Information Technology Education Volume 7, 2008, pp.101-120.

[21] Deborah J. Armstrong , Cynthia K. Riemenschneider, Myria W. Allen , Margaret F. Reid, "Advancement, voluntary turnover and women in IT: A cognitive study of work-family conflict", Information \& Management, 44,2007, pp.142-153.

[22] Janice Anna Knights \& Barbara Jean Kennedy,"Psychological Contract Violation: Impacts on Job Satisfaction and Organizational Commitment Among Australian Senior Public Servants", Applied H.R.M. Research, 2005, Volume 10, Number 2, pp.57-72

[23] Jordan Shropshire \& Christopher Kadlec, "I'm Leaving the IT Field: the Impact of Stress, Job Insecurity, and Burnout on IT Professionals", International Journal of Information and Communication Technology Research, Volume 2 No. 1, January 2012, pp.6-16.

[24] Sarah Beecham, Nathan Baddoo, Tracy Hall, Hugh Robinson \& Helen Sharp, "Motivation in Software Engineering: A systematic literature review", Information and Software Technology 50, 2008, pp.860878.

[25] Jamie Cano \& Greg Miller, "A Gender Analysis of Job Satisfaction, Job Satisfier Factors, and Job Dissatisfier Factors of Agricultural Education Teachers", Journal of Agricultural Education, 1992, pp.40-46.

[26] Omesh Chadha, Employee Satisfaction- A Study Of Hcl Limited, International Journal Of Research In Commerce \& Management, ISSN 0976-2183, Volume No. 2, Issue No. 9,2011, pp. 131-135.

[27] S.M. Shariq Abbas, "Banking Professionals And Attitudinal Dimension Of Job Satisfaction: A Descriptive Study", International Journal Of Research In Commerce, IT \& Management, ISSN 2231-5756, Volume No.1, Issue No. 6, 2011, pp.119-124.

[28] Syed Akif Hasan \& Muhammad Imtiaz Subhani,"Managerial Social Wisdom: A Major Facet for Employee Turnover Intentions, Work Commitments and Manager-Subordinate Relationships, European Journal of Economics, Finance and Administrative Sciences, ISSN 1450-2275, Issue 40,2011, pp.132-137.

[29] Sohrab Ahmad, Khurram Shahzad, Shams-ur-Rehman, Nadeem Ahmed Khan \& Ikram Ullah Shad, "Impact of Organizational Commitment and Organizational Citizenship Behavior on Turnover Intentions of Call Center Personnel in Pakistan", European Journal of Social Sciences - Volume 17, Number 4,2010, pp.585591.
[30] Hamdia Mudor \& Phadett Tooksoon, "Conceptual framework on the relationship between human resource management practices, job satisfaction, and turnover", Journal of Economics and Behavioral Studies, Vol. 2, No. 2, 2011, pp.41-49.

[31] Iqtidar Ali Shah, Zainab Fakhr, M. Shakil Ahmad \& Khalid Zaman, "Measuring Push, Pull and Personal Factors Affecting Turnover Intention: A Case Of University Teachers In Pakistan", Review of Economic \& Business Studies, ISSN-1843-763X, Volume 3, Issue 1, 2010, pp. 167-192.

[32] Priya Chandna \& Venkat R. Krishnan, "Organizational Commitment of Information technology professionals: Role of Transformational leadership and work-related beliefs", Tecnia Journal of Management Studies, Vol. 4 , No. 1, 2009, pp.1-13.

[33] Dr.S.S. Riaz Ahamed,"Studying the feasibility and importance of Software Testing: An Analysis", International Journal of Engineering Science and Technology, Vol.1(3),2009, pp.119-128

[34] Xihui Zhang, Jasbir Dhaliwal \& Mark L. Gillenson," Organizing Software Testing For Improved Quality and Satisfaction", Journal of Information Technology Management, ISSN :1042-1319, Volume XXI, Number 4, 2010, pp. 1-12

[35] Thomas Acton \& Willie Golden ,'Training: The Way to Retain Valuable IT Employees?", Informing Science,2002, pp.1-12

[36] Tariq Mehmood Rana, M. Rashid Salaria, Gobind M. Herani and Mohammad Ahmed Amin, "Identifying Factors Playing Important Role in the Increasing Employee Turnover Rate: A Case of Telecom Industry in Pakistan", Indus Journal of Management \& Social Sciences, 3(2), 2009, pp.80-89.

[37] Caramollah Daneshfard \& Kokab Elsadat Ekvaniyan,Organizational Commitment and Job Satisfaction In Islamic Azad University, Interdisciplinary Journal Of Contemporary Research In Business, Vol.3, No.9,2012, pp.168-181.

[38] Sinem Aydogdu \& Baris Asikgil, "An Empirical Study of the Relationship Among Job Satisfaction,Organizational Commitment and Turnover Intention, International Review of Management and Marketing, ISSN: 2146-4405, Vol. 1, No. 3, 2011, pp.4353.

[39] Raheleh Emami, Ebrahim Moradi, Durrishah Idrus \& Dhaifallah Obaid Almutairi "Investigating the Relationship between Organizational Learning Culture, Job Satisfaction and Turnover Intention in it SMEs", International Journal of Innovative Ideas, Volume 12(1),2012, pp. 8-23.

[40] M.Kannan \& K.Vivekanandan, "The cost and impact of staff attrition", International Journal of Emerging Technologies and Applications in Engineering Technology and Sciences, ISSN: 0974 -3588, Vol.3, Issue 2, 2010, pp.37-40.

[41] M.Kannan \& K.Vivekanandan, "A Study on Attrition", ACCST Research Journal, ISSN: 0972 7779, Vol. VII, No.4, 2009. 